The following comparison of the structure given by Badami and by me for the line $\lambda 5639 \cdot 7$ $\left(6 s{ }^{3} P_{2}-6 p{ }^{3} S_{1}\right)$ shows to what extent the hollow cathode patterns are more clearly resolved:

\begin{tabular}{|c|c|c|c|c|c|c|c|c|}
\hline Badami & $\begin{array}{c}0 \\
(6)\end{array}$ & & $\begin{array}{r}205 \\
(5)\end{array}$ & & & $\begin{array}{l}450 \\
(4)\end{array}$ & $\begin{array}{l}584 \\
(4)\end{array}$ & $\begin{array}{l}780 \\
\text { (1) } \mathrm{cm}^{-1} \times 10^{-3}\end{array}$ \\
\hline Tolans & $\begin{array}{c}0 \\
(10)\end{array}$ & $\begin{array}{l}71 \\
(8)\end{array}$ & 217 & $\begin{array}{r}319 \\
(3)\end{array}$ & $\begin{array}{l}399 \\
(3 \ddagger)\end{array}$ & $\begin{array}{l}477 \\
(6)\end{array}$ & $\begin{array}{r}605 \\
(5)\end{array}$ & $\begin{array}{l}728 \\
\left(1 \frac{1}{2}\right) \mathrm{cm} \cdot .^{-1} \times 10^{-3}\end{array}$ \\
\hline
\end{tabular}

It is seen that Badami's values are those which would arise from the blending of components due to excessive line width.

Full details with analysis will be communicated elsewhere shortly.

$$
\begin{gathered}
\text { Astrophysics Department, } \\
\text { Imperial College of Science, } \\
\text { London, S.W.7. } \\
\text { March 3. }
\end{gathered}
$$

${ }^{1}$ J. S. Badami, Z. Phys., 79, 206 ; 1932.

\section{S. Tolansky.}

\section{The "Neutrino"}

THE view has recently been put forward ${ }^{1}$ that a neutral particle of about electronic mass, and spin $\frac{1}{2} \hbar$ (where $\hbar=h / 2 \pi$ ) exists, and that this 'neutrino' is emitted together with an electron in $\beta$-decay. This assumption allows the conservation laws for energy and angular momentum to hold in nuclear physics ${ }^{2}$. Both the emitted electron and neutrino could be described either $(a)$ as having existed before in the nucleus or (b) as being created at the time of emission. In a recent paper ${ }^{3}$ Fermi has proposed a model of $\beta$. disintegration using $(b)$ which seems to be confirmed by experiment.

According to $(a)$, one should picture the neutron as being built up of a proton, an electron and a neutrino, while if one accepts $(b)$, the rôles of neutron and proton would be symmetrical ${ }^{4}$ and one would expect that positive electrons could also sometimes be created together with a neutrino in nuclear transformations. Therefore the experiments of Curie and Joliots on an artificial positive $\beta$-decay give strong support to method $(b)$, as one can scarcely assume the existence of positive electrons in the nucleus.

Why, then, have positive electrons never been found in the natural $\beta$-decay? This can be explained by the fact that radioactivity usually starts with $\alpha-e m i s s i o n$ and therefore leads to nuclei the charge of which is too small compared with their weight. The artificial $\beta$-emission was found for two unstable nuclei (most probably $\mathrm{N}^{13}$ and $\mathrm{P}^{30}$ ) formed by capture of an $\alpha$-particle and emission of a neutron, and therefore having too high a charge for their mass.

A consequence of assumption (b) is that two isobares differing by 1 in atomic number can only be stable if the difference of their masses is less than the mass of electron and neutrino together. For otherwise the heavier of the two elements would disintegrate with emission of a neutrino and either a positive or negative electron. There will be only a limited region on the mass defect curve, probably at medium atomic weight, where such small differences are possible. In fact, neighbouring isobares have only been found with the mass numbers 87, 115 , $121,123,(187),(203)$, while isobares with atomic numbers differing by 2 are very frequent. In the first case, one of the two nuclei (Rb) is known to emit $\beta$-rays. In each of the last two cases one of the two isobares is stated to be exceedingly rare and its identification might be due to experimental error. The other three cases actually lie close together and have medium weight. A particular case of isobares are proton and neutron. Since all experimentally deduced values of the neutron mass lie between 1.0068 and 1.0078 , they are certainly both stable even if the mass of the neutrino should be zero.

The possibility of creating neutrinos necessarily implies the existence of annihilation processes. The most interesting amongst them would be the follow. ing : a neutrino hits a nucleus and a positive or negative electron is created while the neutrino disappears and the charge of the nucleus changes by 1 .

The cross section $\sigma$ for such processes for a neutrino of given energy may be estimated from the lifetime $t$ of $\beta$-radiating nuclei giving neutrinos of the same energy. (This estimate is in accord with Fermi's model but is more general.) Dimensionally, the connexion will be

$$
\sigma=A / t
$$

where $A$ has the dimension $\mathrm{cm}^{2}$ sec. The longest length and time which can possibly be involved are $\hbar / m c$ and $\hbar / m c^{2}$. Therefore

$$
\sigma<\frac{\hbar^{3}}{m^{3} c^{4} t}
$$

For an energy of $2.3 \times 10^{8}$ volts, $t$ is 3 minutes and therefore $\sigma<10^{-44} \mathrm{~cm}^{2}$ (corresponding to a penetrating power of $10^{16} \mathrm{~km}$. in solid matter). It is therefore absolutely impossible to observe proeesses of this kind with the neutrinos created in nuclear transformations.

With increasing energy, $\sigma$ increases (in Fermi's model $^{3}$ for large energies as $\left.\left(E / m c^{2}\right)^{2}\right)$ but even if one assumes a very steep increase, it seems highly improbable that, even for cosmic ray energies, $\sigma$ becomes large enough to allow the process to be observed.

If, therefore, the neutrino has no interaction with other particles besides the processes of creation and annihilation mentioned-and it is not necessary to assume interaction in order to explain the function of the neutrino in nuclear transformations-one can conclude that there is no practically possible way of observing the neutrino.

$$
\begin{aligned}
& \text { Physical Laboratory, } \\
& \text { University, } \\
& \text { Manchester. }
\end{aligned}
$$

$1 \mathrm{~W}$. Pauli, quoted repeatedly since 1931 , to be published shortly in "Rapports du Septième Conseil Solvay, Brussels", 1933.

C. D. Ellis and N. F. Mott, Proc. Roy. Soc., A, 141, 502 ; 1933. E. Fermi, La Ricerea Scientifica, 2, No. 12 ; 1933.

4 This point of view was flrst put forward by 1 . Curie and F. Joliot at the Conseil Solvay, 1933 .

s. Curie and F. Joliot, NATURE, 133, 201, Feb. 10, 1934.

\section{Changes in the Lipolytic Activity of Different Organs during Tuberculosis}

IN continuation of our earlier work on lipases ${ }^{1}$, we have followed the changes in the lipase content of different organs of guinea pigs infected with bovine tuberculosis. Lipase determinations were made from liver, pancreas, lungs and blood serum, using tributyrine as substrate. It appeared that, coincident 